\title{
Effects of Heparin Therapy on Hexagonal Lipid Neutralization \\ Test
}

Keywords: Heparin; Hexagonal lipid neutralization test; Lupus anticoagulants; Antiphospholipid syndrome; Phospholipid neutralization

\begin{abstract}
In this retrospective study; we evaluate the effects of heparin therapy on hexagonal lipid neutralization test. We identified 8000 patients in our hospital system that had been evaluated for lupus anticoagulants. Among these patients we found 100 patients who had been evaluated for lupus anticoagulants while they were on and off unfractionated (UFH) heparin. $23 \%$ had positive result of hexagona lipid neutralization test before receiving heparin while $59 \%$ had positive result of hexagonal lipid neutralization test after receiving heparin. We conclude that there is an association between heparin therapy and positive result of hexagonal lipid neutralization test.
\end{abstract}

\section{Abbreviations}

LAs: Lupus Anticoagulants; ISTH: International Society of Thrombosis and Haemostasis; PT: Prothrombin Time; TTI: Tissue Thromboplastin Inhibition Index; DRVVT: Dilute Russell Viper Venom Time; HLN: Hexagonal Lipid Neutralization; ACA: Anticardiolipin; a $\beta 2$ GP1: anti $\beta-2$ Glycoprotein I; MARS: Medical Archival Retrieval System; LA: Lupus Anticoagulant

\section{Introduction}

Lupus anticoagulants (LAs) are a heterogeneous class of immunoglobulins that may develop spontaneously or as a consequence of autoimmune diseases. They bind to proteins such as $\beta 2$-glycoprotein $I$, prothrombin, or others in complex with negatively charged phospholipids and thus prolong phospholipiddependent coagulation tests [1]. Clinical interest in antiphospholipid antibodies is due to their relation with arterial and venous thrombosis in the antiphospholipid syndrome. Two forms of antiphospholipid syndrome have been described: a primary syndrome [2] with no evidence of an underlying disease, and a secondary syndrome, mainly in the setting of systemic lupus erythematosus [3]. By consensus definition, a LA is considered present if the patient's plasma exhibits prolongation in a clot-based test, and evidence of a phospholipid dependent of coagulation in the absence of a specific inhibitor. A variety of laboratory tests are available to detect LAs; however, no single test is ideal and each has significant shortcomings. As a result, testing algorithms which incorporate multiple tests expected to be altered by LA are used to establish the diagnosis. The International Society of Thrombosis and Haemostasis (ISTH) has published testing guidelines that include performing two screening tests using different assay principles to demonstrate prolongation of a phospholipid dependent clotting time, a mixing study to evaluate the presence of an inhibitor, and a confirmatory test that demonstrates phospholipid-dependent inhibitory activity in patient plasma [4].
Journal of

Hematology \& Thrombosis

\author{
Abuzar Moradi Tuchayi and Roy E. Smith* \\ Division of Hematology/Oncology, University of Pittsburgh Medical \\ Center, Pittsburgh, PA 15232, USA

\section{${ }^{*}$ Address for Correspondence:} \\ Roy E. Smith, Division of Hematology/Oncology, University of Pittsburgh \\ Medical Center, 5150 Centre Avenue, Pittsburgh, PA 15232, USA, Tel: \\ 412-648-6466; Fax: 412-648-6579; E-mail: smithre@upmc.edu \\ Submission: 19 March, 2016 \\ Accepted: 29 March, 2016 \\ Published: 04 April, 2016 \\ Copyright: (๑) 2016 Tuchayi AM, et al. This is an open access article \\ distributed under the Creative Commons Attribution License, which \\ permits unrestricted use, distribution, and reproduction in any medium, \\ provided the original work is properly cited.
}

Reviewed \& Approved by: Dr. Raul H. Morales-Borges, American Red Cross in San Juan, Practices, Ashford Institute of Hematology \& Oncology, USA

The typical screening test is the APTT which is often prolonged in the presence of a LA. Characteristically, the prolonged APTT fails to correct when the patient's plasma is mixed with an equal amount of normal plasma. However, this screening alone is inadequate to establish the presence of a LA because as many as $20 \%$ of affected patients have normal APTT's. Other tests that aid in the recognition and confirmation of LAs include prothrombin time (PT), tissue thromboplastin inhibition index (TTI), dilute Russell viper venom time (DRVVT), hexagonal lipid neutralization (HLN) test, and testing for anticardiolipin (ACA) and anti $\beta-2$ glycoprotein I (a $\beta 2 \mathrm{GP} 1)$ [57]. The HLN test is performed by mixing a sample of patient plasma with buffer and a second aliquot of the same plasma sample with hexagonal phase phosphatidylethanolamine to neutralize any LA present. Each mixture is then incubated in normal plasma to correct for any coagulation factor deficiency that may be present in the patient's plasma. The APTT of both mixtures is ascertained. A LA is thought present if the confirmatory test is significantly shorter than the screening test. Many clinicians consider the presence of a positive HLN test to be sufficient to confirm the diagnosis of a LA. This is of concern since in our experience the HLN test may frequently be abnormal in the absence of LA and upon exposure to UFH. Because of this observation, we explored the frequency of this abnormality in our patient population.

\section{Materials and Methods}

We performed a retrospective case series study at our multiinstitutional center. This study was approved by our Institutional review board in 06/12/2015. We reviewed 8000 patients identified by the hospital Medical Archival Retrieval System (MARS) laboratory data system who had been evaluated for LAs from January 2011-February 2015. Finally, we found 100 patients who had been evaluated for lupus anticoagulants while they were both receiving and not receiving UFH. We excluded the patients who were receiving other medications (such as the targeted oral anticoagulants) which might interfere with detecting the presence of a LA or who had 
documentation of receiving heparin in the past. We excluded the patients who were suffering from other kinds of coagulopathies. The MARS query included the physical examination, hospital course, and laboratory data.

All patients were evaluated according to our institutional LA panel which consisted standardized methods for the APTT, APTT mixing studies,

TT (16-22 seconds), TTI (Triniclot PT Excel Thromboplastin Reagent, Trinity Biotech, I D A Business Park, Southern Cross, Bray, Co Wicklow, Ireland), DRVVT screen and confirm (Hemosil ACL, Instrumentation Laboratories, 180 Hartwell Road, Bedford, MA 01730), HLN test (Staclot, Diagnostica, Stago, 5 Century Drive, Parsippany, New Jersey 07054), ACA IgG, IgM and IgA (Quanta Lite ELIZA, Inova Diagnostics, 9900 Old Grove Road, San Diego, California 92131), aß2GP1 (Quanta Lite ELIZA, 9900 Old Grove Road, San Diego, California 92131). Residual UFH was eliminated from each plasma sample via the heparin neutralizer included in each test kit or by the addition of heparinase.

After selecting the patients who had been evaluated for Las both while receiving UFH and while not receiving UFH. The patients receiving UFH were not re-evaluated for the presence of a LA for at least fourteen days after the administration of UFH was stopped to eliminate any residual heparin effect.

We collected the demographic information which included age, gender and race. All data were entered into Microsoft excel worksheet and statistical analysis performed using SPSS Version 22. The median, minimum and maximum range was used to describe nonparametric continuous variables such as age. McNemar test was used to assess relationships between groups. Two-sided P-value < 0.05 was designated as significant.

\section{Results}

Among the 100 patients identified, median age was 53 (2097). Thirty eight patients were males. Sixty two patients were females. Eighty two patients were Caucasian and eighteen patients were African American. Just 4 patients were evaluated for APTT mixing studies. The plasma sample obtained from one patient with a prolonged APTT while taking UFH did not correct after mixing and incubation with normal plasma. The plasma sample from one other patient with a prolonged APTT which persisted after cessation of unfractionated heparin therapy did not correct with mixing and incubation with normal plasma. Eighteen patients did not have prolonged APTT while they were on UFH. In all cases, the time from last administration of UFH was greater than 10 minutes and the mean value of the last dose of UFH in these patients was 44.57 units $/ \mathrm{kg}$ (2390). All of the patients had prolonged TT while they were receiving UFH. Eight patients had increased anticardiolipin IgM antibody (13.5-23.8) while receiving UFH and seven patients had increased anticardiolipin IgM antibody (12.8-50.9) when not receiving UFH. Three patients had increased anticardiolipin IgG antibody (16.2-18.5) while receiving UFH and seven patients had increased anticardiolipin IgG antibody (15.6-29.7) when not receiving UFH. No patient had been evaluated for anticardiolipin IgA antibody. One patient had increased anti-beta2 glycoprotein 1 , IgM $(18 \mathrm{U} / \mathrm{ml})$ while receiving
UFH, and one patient had increased anti-beta2 glycoprotein 1, IgM $(52.8 \mathrm{U} / \mathrm{ml})$ after UFH therapy was stopped. No patient had an increased anti-beta2 glycoprotein 1 , IgA while receiving UFH. One patient had an increased anti-beta2 glycoprotein 1, IgA (26.5 U/ $\mathrm{ml}$ ) after UFH was stopped. No patient had an increased anti-beta2 glycoprotein 1, IgG on UFH while one patient had increased antibeta2 glycoprotein $1, \operatorname{IgG}(18.5 \mathrm{U} / \mathrm{ml})$ off UFH. The effect of UFH on hexagonal lipid neutralization test is shown in Figure 1. The effect of UFH on APTT \& TT is shown in Figure 2. The effect of UFH on TTI \& DRVV is shown in Figure 3.

\section{Discussion}

Lupus anticoagulant (LA) testing is frequently ordered when someone has had an unexplained thrombotic episode, has had recurrent miscarriages or late pregnancy complications, particularly in patients with an underlying autoimmune disorder, and/or as a follow-up to a prolonged APTT test. Varied circumstances, including anticoagulant administration frequently interferes with the results

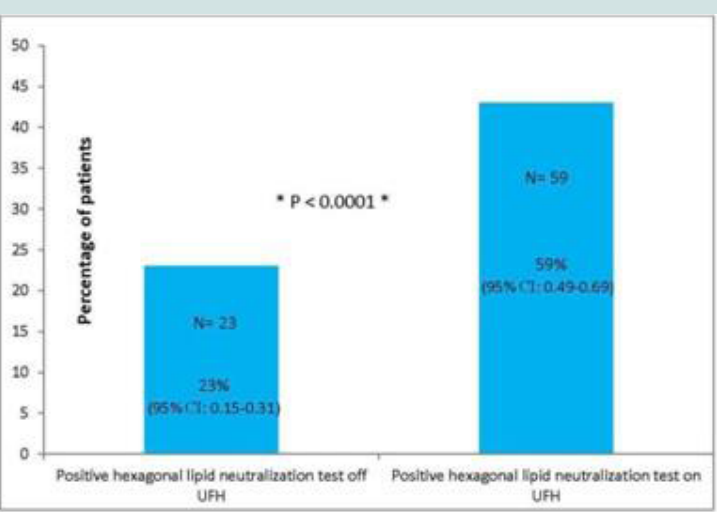

Figure 1: Effects of UFH on Hexagonal lipid neutralization test

The frequency of positive hexagonal lipid neutralization test results in patients which were on UFH $59 \%$ (95\% Cl: $0.49-0.69)$ compared to the frequency of positive hexagonal lipid neutralization test results in patients which were off UFH $23 \%$ (95\% Cl: 0.15-0.31) was statistically significant $\mathrm{P}<0.0001$.

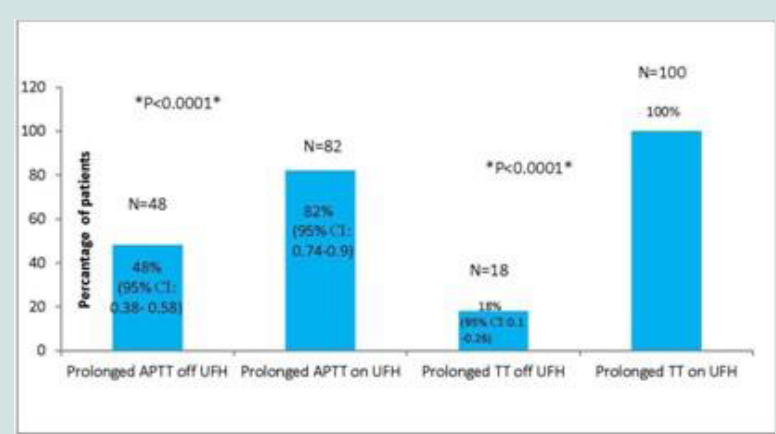

Figure 2: Effects of UFH on APTT \& TT

The frequency of prolonged APTT results in patients which were on UFH $82 \%$ (95\% Cl: 0.74-0.9) compared to the frequency of prolonged APTT results in patients which were off UFH $48 \%(95 \% \mathrm{Cl}$ : $0.38-0.58)$ was statistically significant $\mathrm{P}<0.0001$, and also the frequency of prolonged TT results in patients which were on UFH $100 \%$ compared to the frequency of prolonged TT results in patients which were off UFH $18 \%$ (95\% Cl: $0.1-0.26)$ was statistically significant $\mathrm{P}<0.0001$. 


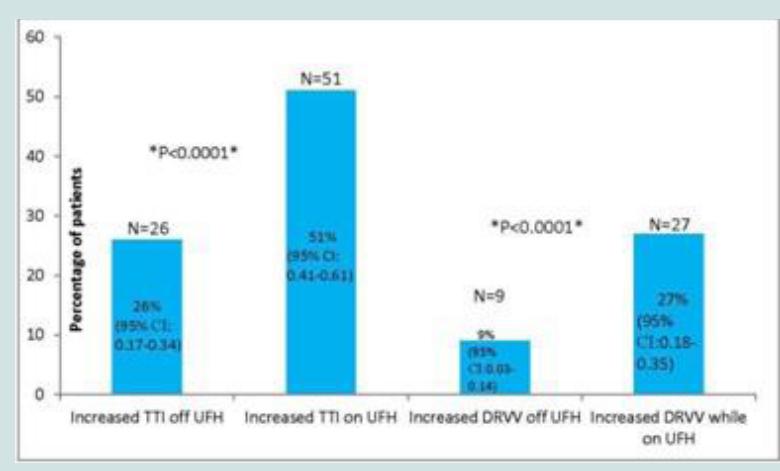

Figure 3: Effects of UFH on TTI \& DRVV

The frequency of increased TTI results in patients which were on UFH $51 \%$ (95\% Cl: 0.41-0.61) compared to the frequency of increased TTI results in patients which were off UFH $26 \%(95 \% \mathrm{Cl}: 0.17-0.34)$ was statistically significant $\mathrm{P}<0.0001$, and also the frequency of increased DRVV results in patients which were on UFH $27 \%(95 \% \mathrm{Cl}$ : 0.18- 0.35) compared to the frequency of increased DRVV results in patients which were off UFH $9 \%$ $(95 \% \mathrm{Cl}: 0.03-0.14)$ was statistically significant $\mathrm{P}<0.0001$

and interpretation of the elements contained in the LA panel. Since patients with thrombosis often require immediate and prolonged anticoagulation, a diagnostic dilemma may occur since heparin therapy may cause a mistaken diagnosis of LAs. It may prolong the APTT and confound the APTT mixing studies and other APTTbased LA tests. Although the TT is often used to exclude the presence of heparin contamination in a plasma sample, it remains uncertain whether normalization of the TT reflects the mitigation of all heparin effects. Heparin neutralizing agents (e.g. heparinase; polybrene) are either added to commercial LA kits or added to patient plasma at the institutional laboratory to obviate the effect of heparin in the specimen. The efficacy of these "in vitro" manipulations of heparin-containing plasma samples are impressive; however, their ability to completely eliminate all biological effects of UFH is inconstant and often requires repeated treatment to obtain satisfactory reversal of anticoagulant activity. Polybrene is a polyamino acid which neutralizes the negatively charged heparin by forming inactive complexes. Heparinase is an enzyme which progressively degrades heparin until its antithrombin binding sites are no longer available. From a practical standpoint, the efficacy of heparin neutralizers is determined by their ability to reverse the heparin-induced prolongation in the APTT [8]. Their effects on other biological activities of UFH are largely unknown. Heparin has also been shown to prolong the DRVVT result [9]. If UFH is not completely neutralized in a plasma sample, it can mimic an LA by recapitulating the first 3 diagnostic laboratory criteria of LA: specifically, prolongation of the APTT, incomplete correction in mixing studies, and a positive hexagonal phospholipid neutralization test or other type of phospholipid neutralization procedure result $[10,11]$. This is particularly the case when the laboratory used intact platelets as a source of phospholipid while performing a phospholipid neutralization procedure.

Evidence indicates that if the patient is taking heparin and the DRVVT is prolonged, the neutralization of the prolonged clotting time by platelet-derived phospholipid may not confirmatory, as large amounts of platelet-derived platelet factor 4 can inhibit the heparin effect $[12,13]$. This observation is particularly important since it demonstrates that UFH has a multiplex effect on plasma components which may not be entirely reflected by a correction in the TT with the addition of heparin neutralizing agents.

Based upon the results presented here, we conclude that there is a strong association between use of UFH and false-positive HLN test results in the absence of a LA. This study indicates that clinicians should view the results of a LA which includes a positive hexagonal lipid neutralization test with caution when the patient is being treated with UFH. Furthermore, the HLN test alone should never be considered an unambiguous indication that a lupus anticoagulant has been detected. This point is emphasized by our observation that heparin neutralizers did not negate the effect of UFH on the HLN test shortly after the administration of UFH was stopped even with a predicted correction in the APTT or thrombin time.

This study has several strengths and weaknesses. Among the strengths are the large number of patients which reduced the play of selection bias and the exclusion of patients who have been switched to warfarin or other newer anticoagulants after cessation of UFH. In addition, since the LA panels were evaluated at least 10 minutes from the cessation of UFH administration, we were able to ascertain that heparin neutralization had a varied effect on clot based tests such as the TT, DRVVT, and activated partial thromboplastin time. Weaknesses include the retrospective design, the initial selection and exclusion of patients which was based upon data derived from the electronic medical record, and the number of patients which had to be excluded from this assessment due to lack of complete data availability. We excluded patients receiving low molecular heparin (LMWH) since during their hospitalization they were concurrently transitioned from LMWH to oral anticoagulation.

UFH exerts its anticoagulant effect by binding to antithrombin [14-16]. We could not assess the role of antithrombin on our findings due to the incompleteness of available data regarding antithrombin activities while the patients with either receiving or not receiving UFH. Due to the retrospective nature of this study, we were unable to establish a heparin dose effect on the HLN test or any effect on measured anticardiolipins.

In conclusion, this retrospective study demonstrates that the hexagonal lipid neutralization test can be falsely positive in patients both while receiving therapeutic doses of UFH and after its cessation despite the addition of heparin neutralizers in the test plasma. For this reason, the interpretation of the HLN test should be undertaken with caution and repeated at a time remote from UFH exposure.

\section{References}

1. Arnout J (2001) Antiphospholipid syndrome: diagnostic aspects of lupus anticoagulants. Thromb haemost 86: 83-91.

2. Asherson RA, Khamashta MA, Ordi-Ros J, Derksen RH, Machin SJ, et al. (1989) The "primary" antiphospholipid syndrome: major clinical and serological features. Medicine (Baltimore) 68: 366-374

3. Alarcon-Segovia D, Deleze M, Oria CV, Sanchez-Guerrero J, GomezPacheco L, et al. (1989) Antiphospholipid antibodies and the antiphospholipid syndrome in systemic lupus erythematosus. A prospective analysis of 500 consecutive patients. Medicine (Baltimore) 68: 353-365.

4. Pengo V, Tripodi A, Reber G, Rand JH, Ortel TL, et al. (2009) Update of the guidelines for lupus anticoagulant detection. Subcommittee on Lupus Anticoagulant/Antiphospholipid Antibody of the Scientific and Standardisation 
Citation: Tuchayi AM, Smith RE. Effects of Heparin Therapy on Hexagonal Lipid Neutralization Test. J Hematol Thromb 2016;2(1): 4.

Committee of the International Society on Thrombosis and Haemostasis. $J$ Thromb Haemost 7: 1737-1740.

5. Smock KJ, Rodgers GM (2009) Laboratory identification of lupus anticoagulants. Am J Hematol 84: 440-442.

6. Pengo V, Biasiolo A, Rampazzo P, Brocco T (1999) dRVVT is more sensitive than KCT or TTI for detecting lupus anticoagulant activity of anti-beta2 glycoprotein I autoantibodies. Thromb Haemost 81: 256-258.

7. Pengo V, Biasiolo A, Fior MG (1995) Autoimmune antiphospholipid antibodies are directed against a cryptic epitope expressed when beta 2-glycoprotein I is bound to a suitable surface. Thromb Haemost 73: 29-34.

8. Jacobsen EM, Trettenes EJ, Wisloff F, Abildgaard U (2006) Detection and quantification of lupus anticoagulants in plasma from heparin treated patients, using addition of polybrene. Thromb J 4: 3

9. Thiagarajan P, Pengo V, Shapiro SS (1986) The use of the dilute Russell viper venom time for the diagnosis of lupus anticoagulants. Blood 68: 869874.

10. Tripodi A, Biasiolo A, Chantarangkul V, Pengo V (2003) Lupus anticoagulant
(LA) testing: performance of clinical laboratories assessed by a national survey using lyophilized affinity-purified immunoglobulin with LA activity. Clin Chem 49: 1608-1614.

11. Brandt JT, Triplett DA, Alving B, Scharrer I (1995) Criteria for the diagnosis of lupus anticoagulants: an update. On behalf of the Subcommittee on Lupus Anticoagulant/Antiphospholipid Antibody of the Scientific and Standardisation Committee of the ISTH. Thromb Haemost 74: 1185-1190.

12. Bick RL, Baker WF (1999) Antiphospholipid syndrome and thrombosis Semin Thromb Hemost 25: 333-350.

13. Kunkel LA (1992) Acquired circulating anticoagulants. Hematol Oncol Clin North Am 6: 1341-1357.

14. Hirsh J (1991) Heparin. N Engl J Med 324: 1565-1574.

15. Lindahl U, Backstrom G, Hook M, Thunberg L, Fransson LA, et al. (1979) Structure of the antithrombin-binding site in heparin. Proc Natl Acad Sci U S A 76: 3198-3202.

16. Choay J, Petitou M, Lormeau JC, Sinay P, Casu B, et al. (1983) Structureactivity relationship in heparin: a synthetic pentasaccharide with high affinity for antithrombin III and eliciting high anti-factor Xa activity. Biochem Biophys Res Commun 116: 492-499.

\section{Acknowledgements}

Competing interests: the authors have no competing interests.

Abuzar Moradi Tuchayi performed the research.

Roy E. Smith designed the research study.

Roy E. Smith contributed essential tools.

Abuzar Moradi Tuchayi analyzed the data.

Roy E. Smith and Abuzar Moradi Tuchayi wrote the paper. 